\title{
Tripartite separability conditions exponentially violated by Gaussian states
}

\author{
E. Shchukin ${ }^{\text {f }}$ and P. van Loock \\ Johannes-Gutenberg University of Mainz, Institute of Physics, Staudingerweg 7, 55128 Mainz
}

\begin{abstract}
Starting with a set of conditions for bipartite separability of arbitrary quantum states in any dimension and expressed in terms of arbitrary operators whose commutator is a $c$-number, we derive a hierarchy of conditions for tripartite separability of continuous-variable three-mode quantum states. These conditions have the form of inequalities for higher-order moments of linear combinations of the mode operators. They enable one to distinguish between all possible kinds of tripartite separability, while the strongest violation of these inequalities is a sufficient condition for genuine tripartite entanglement. We construct Gaussian states for which the violation of our conditions grows exponentially with the order of the moments of the mode operators. By going beyond second moments, our conditions are expected to be useful as well for the detection of tripartite entanglement of non-Gaussian states. We also demonstrate that our conditions can be easily implemented experimentally.
\end{abstract}

PACS numbers: 03.67.Mn, 03.65.Ud, 42.50.Dv

Keywords: continuous-variables; genuine entanglement; multi-partite entanglement conditions; exponential violation

\section{INTRODUCTION}

One of the first algorithmic solutions of the problem of characterizing entangled states was given in [1. The algorithm presented in that work is based on numerical optimization and thus inherits all limitations of the numerics it is based on. Hence there is clearly a desire for more robust and simple analytical entanglement criteria, which may be adapted to specific applications. The most well-known, analytical entanglement criteria are based on a certain positive, but not completely positive map - the partial transposition (PT) of density matrices [2]. For discrete-variable, bipartite two-qubit and qubit-qutrit states 3, as well as for continuous-variable, bipartite Gaussian states split into one versus arbitrarily many modes [4, 5], negative PT (NPT) is necessary and sufficient for entanglement. While NPT is generally only a sufficient entanglement condition, as such, it is still an unambiguous certifier for entanglement.

In the multipartite case, of course, NPT can also serve as a general indicator of inseparability, provided that the richer structure of systems composed of more than two subsystems is taken into account. More specifically, a multipartite state may have NPT with respect to only one particular splitting and have positive PT (PPT) otherwise. While such a scenario is ambiguous for more than two qubits, it would unambiguously identify a non-fully, only partially inseparable Gaussian state of three harmonic oscillators (three optical modes). In fact, arbitrary tripartite, three-mode mixed Gaussian states can be completely classified by five classes ranging from fully inseparable to fully separable states 6 . The fully inseparable class may then be considered the class of genuinely tripartite entangled states. For discrete-variable states

\footnotetext{
* evgeny.shchukin@gmail.com

$\dagger$ loock@uni-mainz.de
}

such as three-qubit states as well as for three modes in a general continuous-variable, mixed state, the situation is more complex, as there are fully inseparable states which can be constructed as convex combinations of different partially separable (biseparable) states. The strongest notion of tripartite entanglement should exclude such biseparable mixtures. The term genuine multipartite entanglement has been used in the literature on continuousvariable entanglement both in the weak and in the strong sense: in Ref. [7, conditions for full inseparability were presented in terms of linear combinations of the position and momentum operators of arbitrarily many modes (applied experimentally for the first time in Ref. [8]), extending the previously given bipartite criteria by Duan et al. [9] to the multipartite case; in the combined theoretical and experimental work of Ref. 10, a stronger version of the tripartite inequalities of Ref. [7] was derived and applied; and Hyllus and Eisert 11] gave a unified treatment including both weak and strong conditions for multipartite entanglement based on a complete knowledge of the covariance matrix of the continuous-variable state in question. All these criteria are based on the second moments of the mode operators, and most of the criteria are also, at least implicity, based on PT (see also Ref. [12]).

Here, starting from a pair of arbitrary operators $\hat{X}$ and $\hat{Y}$ where $[\hat{X}, \hat{Y}]$ is a nonzero $c$-number, we develop a hierarchy of conditions based on $\mathrm{PT}$ which all tripartite biseparable states 13 must satisfy. Each condition is an inequality for higher-order moments of the state under consideration. If a tripartite state violates an inequality of our hierarchy then it is genuinely entangled in the strong sense. Our hierarchy has the remarkable property that the possible violations of our conditions, scaled to the bound for biseparable states, grows exponentially with the order of the moments. We show that there exist pure three-mode Gaussian states which then give an exponential violation with increasing order. Hence such Gaussian states, easily producible with 
squeezed light and beam splitters [14, 15], appear to exhibit the strongest possible form of tripartite entanglement. Moreover, since our criteria are based on higherorder moments, they can also be expected to allow for more sensitive tests of non-Gaussian tripartite entanglement than those based only on second moments. Among other approaches to tripartite entanglement we refer to Refs. [16, 17]. Higher-order moments were already used in Ref. 18 to test whether one group of modes can be separated from the rest. Our conditions here are more powerful in the sense that they are valid for arbitrary mixtures of differently separable states and thus the violation of these conditions is sufficient for genuine tripartite entanglement. The multipartite higher-order moments can be measured experimentally, see Refs. [19, 20].

\section{BIPARTITE CASE}

We start with the following simple lemma, which is usually used for the case of annihilation and creation operators to express antinormally ordered moments in terms of normally ordered ones.

Lemma 1. For any two operators $\hat{X}$ and $\hat{Y}$ whose commutator $[\hat{X}, \hat{Y}]=c$ is a c-number, we have

$$
\hat{X}^{n} \hat{Y}^{m}=\sum_{k=0}^{\min (n, m)} k !\left(\begin{array}{l}
n \\
k
\end{array}\right)\left(\begin{array}{l}
m \\
k
\end{array}\right) c^{k} \hat{Y}^{m-k} \hat{X}^{n-k}
$$

With this lemma we can now prove one of our main results, on which our condition for genuine tripartite entanglement will be based.

Theorem 1. Let operators $\hat{A}$ and $\hat{B}$ act on different degrees of freedom, with the commutator $\left[\hat{B}, \hat{B}^{\dagger}\right]=c>0$ being a positive c-number. Then the inequality

$$
f_{n}(\hat{A}, \hat{B}) \equiv\left\langle\left(\hat{A}^{\dagger}+\hat{B}\right)^{n}\left(\hat{A}+\hat{B}^{\dagger}\right)^{n}\right\rangle \geqslant c^{n} n !
$$

is valid for all bipartite separable quantum states.

If, in addition to the assumption of the theorem, the equality $\left[\hat{A}, \hat{A}^{\dagger}\right]=\left[\hat{B}, \hat{B}^{\dagger}\right]=c$ holds, then the operators $\hat{A}^{\dagger}+\hat{B}$ and $\hat{A}+\hat{B}^{\dagger}$ commute, since in this case we have $\left[\hat{A}^{\dagger}+\hat{B}, \hat{A}+\hat{B}^{\dagger}\right]=\left[\hat{A}^{\dagger}, \hat{A}\right]+\left[\hat{B}, \hat{B}^{\dagger}\right]=0$, and Eq. (2) becomes $\left\langle\left(\left(\hat{A}^{\dagger}+\hat{B}\right)\left(\hat{A}+\hat{B}^{\dagger}\right)\right)^{n}\right\rangle \geqslant n ! c^{n}$. In the bipartite two-mode case, with $\hat{A}=\hat{a}$ and $\hat{B}=-\hat{b}$ being the annihilation operators of the first and the second mode, respectively, we have

$$
2\left(\hat{a}^{\dagger}-\hat{b}\right)\left(\hat{a}-\hat{b}^{\dagger}\right)=\left(\hat{x}_{a}-\hat{x}_{b}\right)^{2}+\left(\hat{p}_{a}+\hat{p}_{b}\right)^{2}=\hat{O}_{\mathrm{EPR}},
$$

where $\hat{x}=\left(\hat{a}+\hat{a}^{\dagger}\right) / \sqrt{2}, \hat{p}=-i\left(\hat{a}-\hat{a}^{\dagger}\right) / \sqrt{2}$, and this inequality can be written as $\left\langle\hat{O}_{\mathrm{EPR}}^{n}\right\rangle \geqslant 2^{n} n$ !, which has been derived in 21] using another approach (with different convention for position and momentum).
Proof. Expanding the left-hand side of Eq. (2) we get

$$
f_{n}(\hat{A}, \hat{B})=\sum_{k, l=0}^{n}\left(\begin{array}{l}
n \\
k
\end{array}\right)\left(\begin{array}{l}
n \\
l
\end{array}\right)\left\langle\hat{A}^{\dagger n-k} \hat{A}^{n-l} \hat{B}^{k} \hat{B}^{\dagger l}\right\rangle .
$$

Using the relation $\langle\hat{A} \hat{B}\rangle_{\mathrm{PT}}=\left\langle\hat{A} \hat{B}^{\dagger}\right\rangle$, which is proven in Appendix A, we have

$$
f_{n}(\hat{A}, \hat{B})=\sum_{k, l=0}^{n}\left(\begin{array}{l}
n \\
k
\end{array}\right)\left(\begin{array}{l}
n \\
l
\end{array}\right)\left\langle\hat{A}^{\dagger n-k} \hat{A}^{n-l} \hat{B}^{l} \hat{B}^{\dagger k}\right\rangle_{\mathrm{PT}} .
$$

One can easily see that this sum can be simplified as

$$
f_{n}(\hat{A}, \hat{B})=\sum_{k=0}^{n}\left(\begin{array}{l}
n \\
k
\end{array}\right)\left\langle\hat{A}^{\dagger n-k}(\hat{A}+\hat{B})^{n} \hat{B}^{\dagger k}\right\rangle_{\mathrm{PT}}
$$

Since $\left[\hat{A}+\hat{B}, \hat{B}^{\dagger}\right]=c$ is a $c$-number, we can use the relation (1) to write the product $(\hat{A}+\hat{B})^{n} \hat{B}^{\dagger k}$ in the "normally-ordered" form:

$$
(\hat{A}+\hat{B})^{n} \hat{B}^{\dagger k}=\sum_{j=0}^{k} j !\left(\begin{array}{l}
n \\
j
\end{array}\right)\left(\begin{array}{l}
k \\
j
\end{array}\right) c^{j} \hat{B}^{\dagger k-j}(\hat{A}+\hat{B})^{n-j}
$$

Substituting this expansion into Eq. (6), after some simplifications we obtain

$$
f_{n}(\hat{A}, \hat{B})=\sum_{i=0}^{n} \frac{n !}{i !}\left(\begin{array}{c}
n \\
i
\end{array}\right) c^{n-i}\left\langle\left(\hat{A}^{\dagger}+\hat{B}^{\dagger}\right)^{i}(\hat{A}+\hat{B})^{i}\right\rangle_{\mathrm{PT}}
$$

For a separable state, the partially transposed state is positive definite, and then the terms on the right-hand side of this equality with $i>0$ are non-negative and we finally get the desired inequality (2) [22].

\section{TRIPARTITE CASE}

Consider the tripartite case with the three parts labeled by the letters $a, b$, and $c$. A state $\varrho$ is called $1 \mid 23$ partially separable if its first part, $a$, can be separated from the other two, $b$ and $c$, i.e., if it can be represented as $\hat{\varrho}=\sum_{i} p_{i} \hat{\varrho}_{a, i} \otimes \hat{\varrho}_{b c, i}$, where $\hat{\varrho}_{a, i}$ are density operators of the $a$-part and $\hat{\varrho}_{b c, i}$ are bipartite density operators of the $b$ and $c$ parts. To test for this kind of separability we introduce the quantity $\mathcal{A}_{1 \mid 23}^{(m)}$ defined as

$$
\mathcal{A}_{1 \mid 23}^{(m)}=\frac{1}{m !}\left\langle\left(\hat{a}^{\dagger}+\hat{b}+\hat{c}\right)^{m}\left(\hat{a}+\hat{b}^{\dagger}+\hat{c}^{\dagger}\right)^{m}\right\rangle .
$$

This quantity can distinguish between 1|23 separable states and all others according to the following proposition.

Proposition 1. The quantity $\mathcal{A}_{1 \mid 23}^{(m)}$ satisfies the inequalities

$$
\mathcal{A}_{1 \mid 23}^{(m)} \geqslant \begin{cases}2^{m} & \text { for all } 1 \mid 23 \text { separable states } \\ 1 & \text { for all states }\end{cases}
$$


For the pure Gaussian state $|\psi\rangle$ with wave function $\psi(\mathbf{x})=\pi^{-3 / 4} \exp \left(-\frac{1}{2} \mathbf{x}^{\mathrm{T}} A \mathbf{x}\right)$, where the matrix is given by $A=\left(\begin{array}{lll}3 & 2 & 2 \\ 2 & 2 & 1 \\ 2 & 1 & 2\end{array}\right)$, we have $\mathcal{A}_{1 \mid 23}^{(m)}=1$ for all $m \geqslant 1$, so this state minimizes all the quantities $\mathcal{A}_{1 \mid 23}^{(m)}, m \geqslant 1$, simultaneously.

Proof. Taking $\hat{A}=\hat{a}$ and $\hat{B}=\hat{b}+\hat{c}$ in Theorem 1 we get the inequality 9a. On the other hand, the operators $\hat{a}^{\dagger}+\hat{c}$ and $\hat{a}+\hat{c}^{\dagger}$ commute with $\hat{b}$ and $\hat{b}^{\dagger}$, and with each other, so we have $\left[\hat{b}+\hat{a}^{\dagger}+\hat{c}, \hat{b}^{\dagger}+\hat{a}+\hat{c}^{\dagger}\right]=1$, and the inequality (9b) follows from Eq. (1). From the same Eq. (1) we have

$$
\begin{aligned}
& \left(\hat{a}^{\dagger}+\hat{b}+\hat{c}\right)^{m}\left(\hat{a}+\hat{b}^{\dagger}+\hat{c}^{\dagger}\right)^{m}=m ! \\
& +\sum_{k=1}^{m}(m-k) !\left(\begin{array}{c}
m \\
k
\end{array}\right)^{2}\left(\hat{a}+\hat{b}^{\dagger}+\hat{c}^{\dagger}\right)^{k}\left(\hat{a}^{\dagger}+\hat{b}+\hat{c}\right)^{k} .
\end{aligned}
$$

It is an easy exercise to verify that $\left(\hat{a}^{\dagger}+\hat{b}+\hat{c}\right)|\psi\rangle=0$, so that we have $\mathcal{A}_{1 \mid 23}^{(m)}=1$ for all $m \geqslant 1$.

It is clear that complete separability, which can be denoted as $1|2| 3$ separability, is a stronger property than just 1|23 partial separability, but the inequalities $(9)$ do not allow us to distinguish between these two cases. To overcome this disadvantage, we introduce the quantities $\mathcal{A}_{2 \mid 13}^{(m)}$ and $\mathcal{A}_{3 \mid 12}^{(m)}$ that are obtained from $\mathcal{A}_{1 \mid 23}^{(m)}$ by an appropriate permutation of $\hat{a}, \hat{b}$, and $\hat{c}$. These quantities satisfy inequalities similar to inequality $(9)$ for the corresponding kinds of partial separability. We then introduce the symmetric sum $S^{(m)}$ defined as

$$
S_{3}^{(m)}=\frac{1}{3}\left(\mathcal{A}_{1 \mid 23}^{(m)}+\mathcal{A}_{2 \mid 13}^{(m)}+\mathcal{A}_{3 \mid 12}^{(m)}\right) .
$$

Our main result is the following theorem about the properties of this sum.

Theorem 2. The sum $S_{3}^{(m)}$ satisfies the following inequality:

$$
S_{3}^{(m)} \geqslant \begin{cases}2^{m} & \text { for fully separable states } \\ \frac{2^{m}+2}{3} & \text { for biseparable states } \\ 1 & \text { for all states }\end{cases}
$$

The condition $S_{3}^{(m)}<\left(2^{m}+2\right) / 3$ is thus sufficient for genuine tripartite entanglement. For any $m \geqslant 1$ there is no physical state with $S_{3}^{(m)}=1$, so the inequality 12c is always the strict inequality $S_{3}^{(m)}>1$. However, there is a family of pure Gaussian states, parametrized with a real parameter $0 \leqslant \xi<1$, for which $S_{3}^{(m)}(\xi) \rightarrow 1$ as $\xi \rightarrow 1$ for all $m \geqslant 1$. In this limit we then have a gap in $S_{3}^{(m)}$ values between biseparable and genuinely entangled states exponentially growing with $m$.
Proof. The inequalities $12 \mathrm{a}$ and $12 \mathrm{c}$ immediately follow from the inequalities (9a) and (9b), respectively. To prove the inequality $(12 \mathrm{~b})$, recall that a state is biseparable if it is a mixture of $1|23,2| 13$ and $3 \mid 12$ partially separable states. For a 1|23 partially separable state the first term in the definition of $S_{3}^{(m)}$ is larger than or equal to $2^{m}$ and the other two terms are bounded by 1 from below, so the inequality $12 \mathrm{~b}$ ) is valid in this case. A similar argument shows that it also holds for all $2 \mid 13$ and $3 \mid 12$ separable states. But then it is also valid for all their mixtures, i.e., for all biseparable states.

If for some $m_{0}$ there is a pure state $\left|\psi_{0}\right\rangle$ with $S_{3}^{\left(m_{0}\right)}=1$, then we must have $\mathcal{A}_{1 \mid 23}^{\left(m_{0}\right)}=\mathcal{A}_{2 \mid 13}^{\left(m_{0}\right)}=\mathcal{A}_{3 \mid 12}^{\left(m_{0}\right)}=1$. From Eq. [10] it then follows that $\left|\psi_{0}\right\rangle$ must be an eigenstate of $\hat{a}^{\dagger}+\hat{b}+\hat{c}, \hat{a}+\hat{b}^{\dagger}+\hat{c}$ and $\hat{a}+\hat{b}+\hat{c}^{\dagger}$ with eigenvalue zero. We show that there is no such common eigenstate even for any two of these three operators. If, on the contrary, $\left|\psi_{0}\right\rangle$ is a zero-eigenstate of $\hat{a}^{\dagger}+\hat{b}+\hat{c}$ and $\hat{a}+\hat{b}^{\dagger}+\hat{c}$, then

$$
\left(\hat{a}^{\dagger}+\hat{b}+\hat{c}\right)\left|\psi_{0}\right\rangle=\left(\hat{a}+\hat{b}^{\dagger}+\hat{c}\right)\left|\psi_{0}\right\rangle=0
$$

and, thus, we have $\left(\hat{a}^{\dagger}-\hat{b}^{\dagger}\right)\left|\psi_{0}\right\rangle=(\hat{a}-\hat{b})\left|\psi_{0}\right\rangle$. In other words, we have $\hat{X}^{\dagger}\left|\psi_{0}\right\rangle=\hat{X}\left|\psi_{0}\right\rangle$, where $\hat{X}=$ $\hat{a}-\hat{b}$ and $\left[\hat{X}, \hat{X}^{\dagger}\right]=2$. From this we get the equality $\left\langle\psi_{0}\left|\hat{X}^{2}\right| \psi_{0}\right\rangle-\left\langle\psi_{0}\left|\hat{X}^{\dagger 2}\right| \psi_{0}\right\rangle=2$, which is contradictory to the fact that the left-hand side is purely imaginary or zero. Hence we have just proved that the equality $S_{3}^{\left(m_{0}\right)}=1$ is impossible and thus the strict inequality $S_{3}^{\left(m_{0}\right)}>1$ is always valid for all pure states. But due to linearity of $S_{3}^{\left(m_{0}\right)}$ this strict inequality is valid for all, including mixed, tripartite quantum states.

To show that the inequalities $S_{3}^{(m)}>1, m \geqslant 1$ are tight we must find a family of states for which $S_{3}^{(m)}$ goes arbitrarily close to 1 . Such a family is given by the pure Gaussian states $\left|\psi_{\xi}\right\rangle$ with wave function

$$
\psi_{\xi}(\mathbf{x})=\pi \sqrt[-3 / 4]{\sqrt[4]{\operatorname{det} A_{\xi}}} \exp \left(-\frac{1}{2} \mathbf{x}^{\mathrm{T}} A_{\xi} \mathbf{x}\right),
$$

where the matrix $A_{\xi}$ is defined as

$$
A_{\xi}=\left(\begin{array}{lll}
1 & \xi & \xi \\
\xi & 1 & \xi \\
\xi & \xi & 1
\end{array}\right)
$$

This matrix is positive definite for $-1 / 2<\xi<1$ and in Appendix B we show that $S_{3}^{(m)}(\xi) \rightarrow 1$ when $\xi \rightarrow 1$.

One can also give an example of a physical state for which the violation of the inequalities (12) grows exponentially, though at a more moderate rate. Consider the so-called GHZ/W states $\left|\varphi_{a}\right\rangle$, which are pure Gaussian states with the matrix

$$
A_{a}=\left(\begin{array}{ccc}
a & e_{-} & e_{-} \\
e_{-} & a & e_{-} \\
e_{-} & e_{-} & a
\end{array}\right),
$$


where, according to Refs. [23 25],

$$
e_{ \pm}=\frac{a^{2}-1 \pm \sqrt{\left(a^{2}-1\right)\left(9 a^{2}-1\right)}}{4 a} .
$$

This matrix is positive-definite for $a>1$. The choice of the off-diagonal elements $e_{-}$is motivated by the desire to have the diagonal elements of the inverse matrix

$$
A_{a}^{-1}=\left(\begin{array}{ccc}
a & e_{+} & e_{+} \\
e_{+} & a & e_{+} \\
e_{+} & e_{+} & a
\end{array}\right)
$$

equal to the diagonal elements of $A_{a}$. The covariance matrix of $\left|\varphi_{a}\right\rangle$ is

$$
\gamma_{a}=\left(\begin{array}{cc}
A_{a}^{-1} & 0 \\
0 & A_{a}
\end{array}\right)
$$

so such a state is symmetric not only with respect to its modes, but up to some extent also with respect to position and momentum operators. In Appendix $\mathrm{C}$ it is shown that for the state of this family with $a=3 / 2$ the violation of our condition grows at least as $1.16^{\mathrm{m}}$.

According to the classification of tripartite Gaussian states from Ref. [6] the states $\left|\psi_{\xi}\right\rangle$ and $\left|\varphi_{a}\right\rangle$ belong to class 1 , the class of states which are not separable for any grouping of the parties. A tripartite Gaussian state belongs to class 1 if the partially transposed matrices no longer satisfy the physicality condition, i.e., if $\Lambda_{1} \gamma \Lambda_{1} \ngtr i J, \Lambda_{2} \gamma \Lambda_{2} \ngtr i J$ and $\Lambda_{3} \gamma \Lambda_{3} \ngtr i J$, where $\Lambda_{1}=\operatorname{diag}(1,1,1,-1,1,1), \Lambda_{2}=\operatorname{diag}(1,1,1,1,-1,1)$, $\Lambda_{3}=\operatorname{diag}(1,1,1,1,1,-1)$ and

$$
J=\left(\begin{array}{cc}
0 & -\mathbb{1} \\
\mathbb{1} & 0
\end{array}\right) .
$$

Since the state $\left|\psi_{\xi}\right\rangle$ is symmetric, it is enough to test only one of the three conditions. We show that $\Lambda_{1} \gamma \Lambda_{1} \ngtr i J$. The covariance matrix $\gamma_{\xi}$ of the state $\left|\psi_{\xi}\right\rangle$ reads as

$$
\gamma_{\xi}=\left(\begin{array}{cc}
A_{\xi}^{-1} & 0 \\
0 & A_{\xi}
\end{array}\right)
$$

The leading principal minor of the matrix $\Lambda_{1} \gamma_{\xi} \Lambda_{1}-i J$ of size 5 is equal to $-4 \xi^{2} /(1-\xi)^{2}(1+2 \xi)<0$ and thus the condition $\Lambda_{1} \gamma \Lambda_{1} \ngtr i J$ is fulfilled. The same minor of the original matrix $\gamma_{\xi}-i J$ equals to zero, so there is no violation of the physicality condition, as it must be. The principal minor of $\Lambda_{1} \gamma_{a} \Lambda_{1}-i J$ for $a=1.5$ obtained by removing the second row and column is equal to $-1.185<0$ and thus $\left|\varphi_{a}\right\rangle$ also belongs to the class 1 . In fact, in the case of a pure state, one could verify its non-separability directly from its wave function.

\section{CONDITIONS IN TERMS OF POSITION AND MOMENTUM OPERATORS}

The lowest-order quantity $S_{3}^{(1)}$ can be easily expressed in terms of the position and momentum operators:

$$
\begin{aligned}
& S_{3}^{(1)}=(1 / 6) T_{3}^{(1)}+(1 / 2), \text { where } \\
& \begin{aligned}
T_{3}^{(1)}= & 3\left\langle\left(\hat{x}_{a}+\hat{x}_{b}+\hat{x}_{c}\right)^{2}\right\rangle+\left\langle\left(-\hat{p}_{a}+\hat{p}_{b}+\hat{p}_{c}\right)^{2}\right\rangle \\
& +\left\langle\left(\hat{p}_{a}-\hat{p}_{b}+\hat{p}_{c}\right)^{2}\right\rangle+\left\langle\left(\hat{p}_{a}+\hat{p}_{b}-\hat{p}_{c}\right)^{2}\right\rangle .
\end{aligned}
\end{aligned}
$$

The inequalities 12 for $m=1$ can be translated to the following inequalities for $T_{3}^{(1)}$ :

$$
T_{3}^{(1)} \geqslant \begin{cases}9 & \text { for fully separable states } \\ 5 & \text { for biseparable states } \\ 3 & \text { for all states }\end{cases}
$$

Note that the strict inequality $T_{3}^{(1)}>3$ is valid for any quantum state since $S_{3}^{(1)}>1$ holds true. We now derive the inequalities (23) directly from the uncertainty relation.

The left-hand side of these inequalities is the sum of the three quantities $\left\langle\left(\hat{x}_{a}+\hat{x}_{b}+\hat{x}_{c}\right)^{2}+\left(-\hat{p}_{a}+\hat{p}_{b}+\hat{p}_{c}\right)^{2}\right\rangle$, $\left\langle\left(\hat{x}_{a}+\hat{x}_{b}+\hat{x}_{c}\right)^{2}+\left(\hat{p}_{a}-\hat{p}_{b}+\hat{p}_{c}\right)^{2}\right\rangle$ and $\left\langle\left(\hat{x}_{a}+\hat{x}_{b}+\hat{x}_{c}\right)^{2}+\right.$ $\left.\left(\hat{p}_{a}+\hat{p}_{b}-\hat{p}_{c}\right)^{2}\right\rangle$. Each quantity can be estimated with the inequality $\left\langle\hat{X}^{2}+\hat{Y}^{2}\right\rangle \geqslant|\langle[\hat{X}, \hat{Y}]\rangle|$, which is valid for arbitrary observables $\hat{X}$ and $\hat{Y}$. This inequality follows from the positivity of $\langle(\hat{X} \pm i \hat{Y})(\hat{X} \mp i \hat{Y})\rangle \geqslant 0$. From this we immediately obtain the inequality

$$
\left\langle\left(\hat{x}_{a}+\hat{x}_{b}+\hat{x}_{c}\right)^{2}\right\rangle+\left\langle\left(-\hat{p}_{a}+\hat{p}_{b}+\hat{p}_{c}\right)^{2}\right\rangle \geqslant 1,
$$

and similar inequalities for the other two quantities, so 23c follows.

If a state has some separability properties then we can obtain a better estimation. Note that it is enough to prove inequalities 23a and (23b) only for the corresponding factorizable states and then validity of these inequalities for general separable states will follow by linearity of the average. For a 1|23-factorizable state we have

$$
\begin{aligned}
\left\langle\left(\hat{x}_{a}+\hat{x}_{b}+\hat{x}_{c}\right)^{2}\right\rangle & =\left\langle\left(\Delta \hat{x}_{a}\right)^{2}+\left(\Delta \hat{x}_{b}+\Delta \hat{x}_{c}\right)^{2}\right\rangle \\
& +\left\langle\hat{x}_{a}+\hat{x}_{b}+\hat{x}_{c}\right\rangle^{2} \\
\left\langle\left(-\hat{p}_{a}+\hat{p}_{b}+\hat{p}_{c}\right)^{2}\right\rangle & =\left\langle\left(\Delta \hat{p}_{a}\right)^{2}+\left(\Delta \hat{p}_{b}+\Delta \hat{p}_{c}\right)^{2}\right\rangle \\
& +\left\langle-\hat{p}_{a}+\hat{p}_{b}+\hat{p}_{c}\right\rangle^{2} .
\end{aligned}
$$

Since $\left\langle\left(\Delta \hat{x}_{a}\right)^{2}+\left(\Delta \hat{p}_{a}\right)^{2}\right\rangle \geqslant 1$ and

$$
\left\langle\left(\Delta \hat{x}_{b}+\Delta \hat{x}_{c}\right)^{2}+\left(\Delta \hat{p}_{b}+\Delta \hat{p}_{c}\right)^{2}\right\rangle \geqslant 2,
$$

we get

$$
\left\langle\left(\hat{x}_{a}+\hat{x}_{b}+\hat{x}_{c}\right)^{2}\right\rangle+\left\langle\left(-\hat{p}_{a}+\hat{p}_{b}+\hat{p}_{c}\right)^{2}\right\rangle \geqslant 3 .
$$

The other two quantities can be estimated either as we did in the previous paragraph or using this alternative approach. Both lead to the same bound, 1, so we arrive at inequality 23b.

For a completely factorizable state we have the identity

$$
\begin{aligned}
\left\langle\left(\hat{x}_{a}+\hat{x}_{b}+\hat{x}_{c}\right)^{2}\right\rangle & =\left\langle\left(\Delta \hat{x}_{a}\right)^{2}+\left(\Delta \hat{x}_{b}\right)^{2}+\left(\Delta \hat{x}_{c}\right)^{2}\right\rangle \\
& +\left\langle\hat{x}_{a}+\hat{x}_{b}+\hat{x}_{c}\right\rangle^{2}
\end{aligned}
$$


and similar identities for the combinations of $\hat{p}$ 's. We thus have

$$
\left\langle\left(\hat{x}_{a}+\hat{x}_{b}+\hat{x}_{c}\right)^{2}\right\rangle+\left\langle\left(-\hat{p}_{a}+\hat{p}_{b}+\hat{p}_{c}\right)^{2}\right\rangle \geqslant 3
$$

and similar inequalities for the other two quantities, which establishes inequality 23a.

We now demonstrate that the state $\left|\psi_{\xi}\right\rangle$ in the limit $\xi \rightarrow 1$ perfectly violates the inequality 23c. One can easily see that

$$
\hat{\mathbf{p}}\left|\psi_{\xi}\right\rangle=i A_{\xi} \mathbf{x}\left|\psi_{\xi}\right\rangle
$$

This means that the state $\left|\psi_{\xi}\right\rangle$ is a simultaneous eigenstate with zero eigenvalue of the non-Hermitian operators $\hat{p}_{1}-i \hat{x}_{1}-i \xi \hat{x}_{2}-i \xi \hat{x}_{3}, \hat{p}_{2}-i \xi \hat{x}_{1}-i \hat{x}_{2}-i \xi \hat{x}_{3}$, and $\hat{p}_{3}-i \xi \hat{x}_{1}-i \xi \hat{x}_{2}-i \hat{x}_{3}$. In the limit $\xi \rightarrow 1$ the (unphysical) limiting state $\left|\psi_{1}\right\rangle$ is a simultaneous eigenstate with eigenvalue zero of the non-Hermitian operators $\hat{p}_{k}-i \hat{X}$, $k=1,2,3, \hat{X}=\hat{x}_{1}+\hat{x}_{2}+\hat{x}_{3}$. One can show that for such an eigenstate one has $T_{3}^{(1)}=3$, i.e. such an eigenstate perfectly violates the inequality 23c and thus the inequality (12c) (which is impossible for any physical state). In fact, in the limit $\xi \rightarrow 1$ we have the relations

$$
\hat{p}_{1}\left|\psi_{1}\right\rangle=\hat{p}_{2}\left|\psi_{1}\right\rangle=\hat{p}_{3}\left|\psi_{1}\right\rangle=i \hat{X}\left|\psi_{1}\right\rangle
$$

From these equalities we get $T_{3}^{(1)}=6\left\langle\hat{X}^{2}\right\rangle$. To compute the last average, note that

$$
\hat{p}_{1}^{2}\left|\psi_{1}\right\rangle=-\hat{X}^{2}\left|\psi_{1}\right\rangle+\left|\psi_{1}\right\rangle
$$

In a similar way one obtains that $\left\langle\hat{p}_{j} \hat{p}_{k}\right\rangle=-\left\langle\hat{X}^{2}\right\rangle+1$ for all $j, k=1,2,3$. Then, on the one hand we have

$$
\sum_{j, k=1}^{3}\left\langle\hat{p}_{j} \hat{p}_{k}\right\rangle=\left\langle\left(\hat{p}_{1}+\hat{p}_{2}+\hat{p}_{3}\right)^{2}\right\rangle=9\left\langle\hat{X}^{2}\right\rangle .
$$

On the other hand, we have $\sum_{j, k=1}^{3}\left\langle\hat{p}_{j} \hat{p}_{k}\right\rangle=-9\left\langle\hat{X}^{2}\right\rangle+9$. Comparing both equalities, we obtain $\left\langle\hat{X}^{2}\right\rangle=1 / 2$ and thus $T_{3}^{(1)}=3$.

\section{EXPERIMENT}

As it is written, the left-hand side of the inequalities (12) (or, equivalently, the quantity 22 and its higherorder extensions) cannot be measured straightforwardly. In Eq. 22 the combinations of moments commute with each other, but do not commute with the combination of positions, so these four quantities cannot be measured simultaneously. We show now that with little modification of the inequalities 12 it is possible to avoid this drawback.

Let us augment our tripartite system with an auxiliary fourth mode, $z$, which we will assume separable from the first three, i.e., we consider fourpartite states of the form $\hat{\varrho}^{\prime}=\sum_{i} p_{i} \hat{\varrho}_{a b c, i} \otimes \hat{\varrho}_{z, i}$. The quantity $\mathcal{A}_{1 \mid 23}^{(m)}$, defined by Eq. (8), is generalized in the following way:

$$
\mathcal{A}_{1 \mid 23}^{\prime(m)}=\frac{1}{m !}\left\langle\left(\hat{a}^{\dagger}+\hat{b}+\hat{c}+\hat{z}^{\dagger}\right)^{m}\left(\hat{a}+\hat{b}^{\dagger}+\hat{c}^{\dagger}+\hat{z}\right)^{m}\right\rangle .
$$

Taking $\hat{A}=\hat{a}+\hat{z}$ and $\hat{B}=\hat{b}+\hat{c}$ in 2 we obtain that if all $\hat{\varrho}_{a b c, i}$ are $1 \mid 23$-separable then $\mathcal{A}_{1 \mid 23}^{\prime(m)} \geqslant 2^{m}$. On the other hand, if states $\hat{\varrho}_{a b c, i}$ are arbitrary, we can take $\hat{A}=$ $\hat{z}$ and $\hat{B}=\hat{a}^{\dagger}+\hat{b}+\hat{c}$ to conclude that $\mathcal{A}_{1 \mid 23}^{\prime(m)} \geqslant 1$ in this case. We see that $\mathcal{A}_{1 \mid 23}^{\prime(m)}$ satisfies the inequalities 9 under the assumption that the fourth mode is separable from the first three. Moreover, if $\hat{\varrho}^{\prime}=\hat{\varrho} \otimes|0\rangle\langle 0|$, then $\mathcal{A}_{1 \mid 23}^{\prime(m)}=\mathcal{A}_{1 \mid 23}^{(m)}$, so if we can easily measure $\mathcal{A}_{1 \mid 23}^{\prime(m)}$ we can also easily measure $\mathcal{A}_{1 \mid 23}^{(m)}$.

From the inequalities for the augmented quantities $\mathcal{A}_{1 \mid 23}^{\prime(1)}, \mathcal{A}_{2 \mid 13}^{\prime(1)}$ and $\mathcal{A}_{3 \mid 12}^{\prime(1)}$ we can obtain an inequality in terms of the position and momentum operators similar to the inequality 23 . It reads as follows:

$$
T_{3}^{\prime(1)}-3 \geqslant \begin{cases}9 & \text { for fully separable states } \\ 5 & \text { for biseparable states } \\ 3 & \text { for all states }\end{cases}
$$

where $T_{3}^{\prime(1)}$ is defined by

$$
\begin{aligned}
T_{3}^{\prime(1)} & =3\left\langle\left(\hat{x}_{a}+\hat{x}_{b}+\hat{x}_{c}+\hat{x}_{z}\right)^{2}\right\rangle+\left\langle\left(\hat{p}_{a}-\hat{p}_{b}-\hat{p}_{c}+\hat{p}_{z}\right)^{2}\right\rangle+\left\langle\left(-\hat{p}_{a}+\hat{p}_{b}-\hat{p}_{c}+\hat{p}_{z}\right)^{2}\right\rangle+\left\langle\left(-\hat{p}_{a}-\hat{p}_{b}+\hat{p}_{c}+\hat{p}_{z}\right)^{2}\right\rangle \\
& =\left\langle\left(\hat{X}_{a z}+\hat{X}_{b c}\right)^{2}+\left(\hat{P}_{a z}-\hat{P}_{b c}\right)^{2}+\left(\hat{X}_{b z}+\hat{X}_{a c}\right)^{2}+\left(\hat{P}_{b z}-\hat{P}_{a c}\right)^{2}+\left(\hat{X}_{c z}+\hat{X}_{a b}\right)^{2}+\left(\hat{P}_{c z}-\hat{P}_{a b}\right)^{2}\right\rangle
\end{aligned}
$$

with $\hat{X}_{i j}=\hat{x}_{i}+\hat{x}_{j}$ and $\hat{P}_{i j}=\hat{p}_{i}+\hat{p}_{j}$, while the separability test is applied to the first three modes (since the fourth mode is always assumed to be separable from the rest). For a fully separable tripartite state the corresponding four-partite state is also fully separable and we can apply the result of Ref. [9] (scaled by a factor of 2 since we have a sum of two operators) to get that $\left\langle\left(\hat{X}_{i j}+\hat{X}_{k l}\right)^{2}+\left(\hat{P}_{i j}-\hat{P}_{k l}\right)^{2}\right\rangle \geqslant 4,\{i, j, k, l\}=$ $\{a, b, c, z\}$, and thus the inequality $35 \mathrm{a}$ is obtained. If $\hat{X}_{i j}$ and $\hat{X}_{k l}$ were ordinary single-mode operators then $\left\langle\left(\hat{X}_{i j}+\hat{X}_{k l}\right)^{2}+\left(\hat{P}_{i j}-\hat{P}_{k l}\right)^{2}\right\rangle$ could be arbitrarily close to zero, but in our case each operator $\hat{X}_{i j}$ acts on two modes and the fact that one of the modes is always separable from the rest puts a lower bound on the values that this quantity can take. This restriction is expressed 
by $\left\langle\left(\hat{X}_{i j}+\hat{X}_{k l}\right)^{2}+\left(\hat{P}_{i j}-\hat{P}_{k l}\right)^{2}\right\rangle \geqslant 2$, so we arrive at the inequalities (35b) and (35c).

The advantage of introducing a new mode is that all the quantities in Eq. (36) commute with each other and thus can be measured simultaneously. In Fig. 1 we present a scheme to measure $T_{3}^{\prime(1)}$, defined by Eq. (36). It consists of four 50-50 beam splitters and two mirrors. The first three inputs are used for the tripartite state in question and the auxiliary fourth input is vacuum. On the output we get precisely the four combinations from Eq. (36). The higher-order quantities can be measured with a generalization of the approach proposed in Ref. 26].

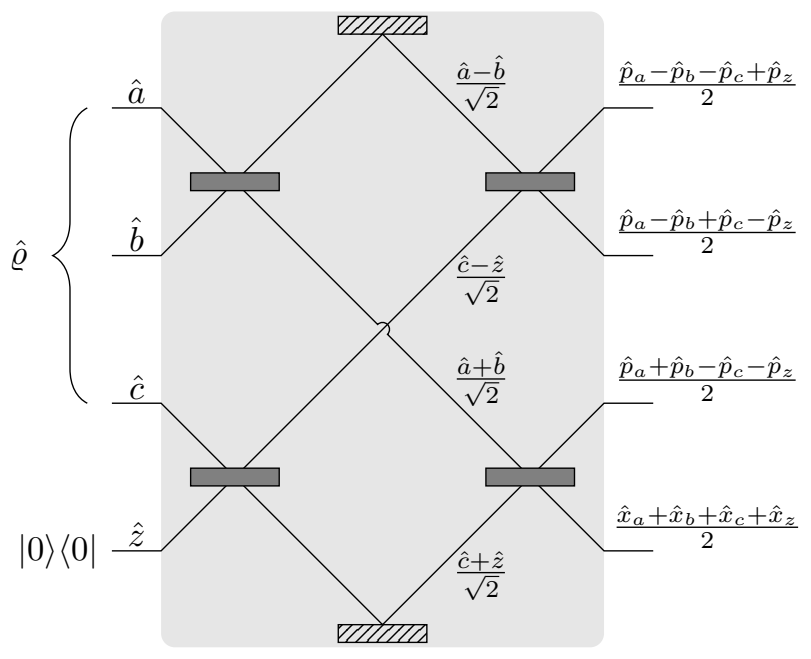

FIG. 1. Measurement scheme for $T_{3}^{\prime(1)}$, Eq. 36.

\section{CONCLUSION}

In conclusion, we have derived a hierarchy of conditions for tripartite separability in terms of higher-order moments. Violation of these conditions is a clear signature of tripartite genuine entanglement. We have constructed tripartite Gaussian states for which the violation of the conditions grows exponentially with the order of the moments. Higher-order conditions of our hierarchy can potentially be useful to detect entanglement of non-Gaussian states as well. We also proposed a method to implement the measurements of our conditions in a straightforward way.

\section{Appendix A: Moments of partially transposed state}

We first prove the relation $\langle\hat{A} \hat{B}\rangle_{\mathrm{PT}}=\left\langle\hat{A} \hat{B}^{\dagger}\right\rangle$, which we use to prove Theorem 1 . For a density operator

$$
\hat{\varrho}=\sum_{n, m, k, l} \varrho_{n m k l}|n\rangle_{1}\langle k|\otimes| m\rangle_{2}\langle l|
$$

its partial transposition with respect to the second subsystem reads as

$$
\hat{\varrho}^{\mathrm{PT}}=\sum_{n, m, k, l} \varrho_{n m k l}|n\rangle_{1}\langle k|\otimes| l\rangle_{2}\langle m| .
$$

In these expressions the states $\left\{|n\rangle_{1}\right\}$ form a basis of the Hilbert space associated with the first subsystem and similarly $\left\{|m\rangle_{2}\right\}$ is a basis of the second subsystem. We then have

$$
\langle\hat{A} \hat{B}\rangle_{\mathrm{PT}}=\sum_{n, m, k, l} \varrho_{n m k l}\langle k|\hat{A}| n\rangle\langle m|\hat{B}| l\rangle .
$$

On the other hand, we can write

$$
\begin{aligned}
\left\langle\hat{A} \hat{B}^{\dagger}\right\rangle & =\sum_{n, m, k, l} \varrho_{n m k l}\langle k|\hat{A}| n\rangle\left\langle l\left|\hat{B}^{\dagger}\right| m\right\rangle \\
& =\sum_{n, m, k, l} \varrho_{n m k l}\langle k|\hat{A}| n\rangle\langle m|\hat{B}| l\rangle=\langle\hat{A} \hat{B}\rangle_{\mathrm{PT}} .
\end{aligned}
$$

Here we have used an additional assumption that the matrix elements $\langle m|\hat{B}| l\rangle$ are real in the basis $\left\{|m\rangle_{2}\right\}$. In the present work we deal with creation and annihilation operators in the Fock basis, so this assumption is valid in our case.

\section{Appendix B: Proof of Theorem 2}

Here we prove that the inequalities $S_{3}^{(m)}>1$ are tight, i.e., one cannot improve the lower bound. Let us take an arbitrary real tripartite Gaussian state $|\psi\rangle$ symmetric with respect to its modes. The matrix $A$ of this state corresponds to

$$
A=\left(\begin{array}{lll}
\mu & \nu & \nu \\
\nu & \mu & \nu \\
\nu & \nu & \mu
\end{array}\right),
$$

where $\mu$ and $\nu$ are some real numbers such that $A$ is positive-definite. Due to symmetry all three terms in the definition of $S_{3}^{(m)}$ are equal and we have

$$
S_{3}^{(m)}=\frac{1}{m !}\left\langle\left(\hat{a}^{\dagger}+\hat{b}+\hat{c}\right)^{m}\left(\hat{a}+\hat{b}^{\dagger}+\hat{c}^{\dagger}\right)^{m}\right\rangle,
$$

so it is enough to compute only one of the terms. We define $\hat{Z}=\hat{a}+\hat{b}^{\dagger}+\hat{c}^{\dagger}$. To find $\hat{Z}^{m}|\psi\rangle$ we first compute the generating function $e^{t \hat{Z}}|\psi\rangle$ and then take the coefficient of $t^{m}$. In the position representation the operator $\hat{Z}$ becomes

$$
\begin{aligned}
\hat{Z} & =\frac{1}{\sqrt{2}}\left(x+y+z+\frac{\partial}{\partial x}-\frac{\partial}{\partial y}-\frac{\partial}{\partial z}\right) \\
& =\frac{1}{\sqrt{2}}\left(\mathbf{j}^{\mathrm{T}} \mathbf{x}+\mathbf{e}^{\mathrm{T}} \nabla\right),
\end{aligned}
$$

where we use the notation $\mathbf{j}=(1,1,1), \mathbf{e}=(1,-1,-1)$ and $\nabla=(\partial / \partial x, \partial / \partial y, \partial / \partial z)$. The exponent of $\hat{Z}$ can 
be easily disentangled with help of the Baker-CampbellHausdorff formula [27, and we have the following result:

$$
e^{t \hat{Z}}=e^{-t^{2} / 4} e^{(t / \sqrt{2}) \mathbf{j}^{\mathrm{T}} \mathbf{x}} e^{(t / \sqrt{2}) \mathbf{e}^{\mathrm{T}} \nabla} .
$$

The Taylor expansion formula can be compactly written in the following symbolic form:

$$
e^{\mathbf{v}^{\mathrm{T}} \nabla} f(\mathbf{x})=f(\mathbf{x}+\mathbf{v}),
$$

so that we can easily compute $\left\langle\mathbf{x}\left|e^{t \hat{Z}}\right| \psi\right\rangle$ as

$$
\begin{aligned}
\left\langle\mathbf{x}\left|e^{t \hat{Z}}\right| \psi\right\rangle & =e^{-\frac{t^{2}}{4}+\frac{t}{\sqrt{2}} \mathbf{j}^{\mathrm{T}} \mathbf{x}} \psi\left(\mathbf{x}+\frac{t}{\sqrt{2}} \mathbf{e}\right) \\
& =e^{-\frac{\lambda}{4} t^{2}+\frac{t}{\sqrt{2}} \mathbf{u}^{\mathrm{T}} \mathbf{x}} \psi(\mathbf{x}),
\end{aligned}
$$

where $\lambda=1+\mathbf{e}^{\mathrm{T}} A \mathbf{e}$ and $\mathbf{u}=\mathbf{j}-A \mathbf{e}$. From this we immediately get an explicit expression for $\hat{Z}^{m}|\psi\rangle$,

$$
\left\langle\mathbf{x}\left|\hat{Z}^{m}\right| \psi\right\rangle=\frac{\sqrt{\lambda^{m}}}{2^{m}} H_{m}\left(\frac{\mathbf{u}^{\mathrm{T}} \mathbf{x}}{\sqrt{2 \lambda}}\right) \psi(\mathbf{x}) .
$$

We then have the following expression for our quantity:

$$
\begin{aligned}
S_{3}^{(m)} & =\frac{1}{m !}\left\langle\hat{Z}^{\dagger m} \hat{Z}^{m}\right\rangle \\
& =\frac{\lambda^{m}}{m ! 2^{2 m}} \int H_{m}^{2}\left(\frac{\mathbf{u}^{\mathrm{T}} \mathbf{x}}{\sqrt{2 \lambda}}\right) \psi^{2}(\mathbf{x}) d \mathbf{x} .
\end{aligned}
$$

To compute this integral, we introduce another generating function

$$
G(t)=\sum_{m=0}^{+\infty} S_{3}^{(m)} t^{m}
$$

With the help of the relation 28.

$$
\sum_{m=0}^{+\infty} \frac{(s / 2)^{m}}{m !} H_{m}^{2}(x)=\frac{1}{\sqrt{1-s^{2}}} e^{\frac{2 s}{1+s} x^{2}}
$$

we can write $G(t)$ in the following form:

$$
G(t)=\frac{1}{\sqrt{1-\frac{\lambda^{2}}{4} t^{2}}} \sqrt{\frac{\operatorname{det} A}{\operatorname{det} A^{\prime}}},
$$

where $A^{\prime}$ is a rank-one update of the matrix $A$

$$
A^{\prime}=A-\frac{1}{2} \frac{t}{1+\frac{\lambda}{2} t} \mathbf{u u}^{\mathrm{T}} \text {. }
$$

For the determinant of this new matrix we have 29]

$$
\operatorname{det} A^{\prime}=\operatorname{det} A\left(1-\frac{1}{2} \frac{t}{1+\frac{\lambda}{2} t} \mathbf{u}^{\mathrm{T}} A^{-1} \mathbf{u}\right),
$$

and then we finally get an explicit expression for the generating function

$$
G(t)=\frac{1}{\sqrt{1-\frac{1+\mathbf{e}^{\mathrm{T}} A \mathbf{e}}{2}} t} \frac{1}{\sqrt{1-\frac{1+\mathbf{j}^{\mathrm{T}} A^{-1} \mathbf{j}}{2}} t} .
$$

We can now prove the rest of Theorem 2 . In the case of the state $\left|\psi_{\xi}\right\rangle$ we have $A \equiv A_{\xi}=\left(\begin{array}{ccc}1 & \xi & \xi \\ \xi & 1 & \xi \\ \xi & \xi & 1\end{array}\right)$, and $1+$ $\mathbf{e}^{\mathrm{T}} A \mathbf{e}=4-2 \xi$ and $1+\mathbf{j}^{\mathrm{T}} A^{-1} \mathbf{j}=\frac{4+2 \xi}{1+2 \xi}$, so that

$$
G_{\xi}(t)=\frac{1}{\sqrt{1-(2-\xi) t}} \frac{1}{\sqrt{1-\frac{2+\xi}{1+2 \xi} t}} .
$$

In the limit $\xi \rightarrow 1$ we immediately obtain

$$
G_{1}(t)=\frac{1}{1-t}=1+t+t^{2}+t^{3}+\ldots,
$$

which means that $\lim _{\xi \rightarrow 1} S_{3}^{(m)}(\xi)=1$.

One can immediately see that our conditions can also be violated for mixed non-Gaussian states of the form $(1-p)\left|\psi_{\xi}\right\rangle\left\langle\psi_{\xi}|+p| \operatorname{vac}\right\rangle\langle\mathrm{vac}|$. The conditions $S_{3}^{(m)} \geqslant$ $\left(2^{m}+2\right) / 3$ are violated for $p<1 / 3$ in the limit $\xi \rightarrow 1$. This means that if the vacuum noise is not so strong $(p<1 / 3)$ then our conditions still work.

By analogy with $\mathcal{A}_{1 \mid 23}^{(m)}$ we can introduce a similar quantity $\tilde{\mathcal{A}}_{1 \mid 23}^{(m)}=\left\langle\left(-\hat{a}^{\dagger}+\hat{b}+\hat{c}\right)^{m}\left(-\hat{a}+\hat{b}^{\dagger}+\hat{c}^{\dagger}\right)^{m}\right\rangle$ and the corresponding symmetric sum $\tilde{S}_{3}^{(m)}$. This new quantity also satisfies the inequalities (12). In terms of position and momentum operators $\tilde{T}_{3}^{(1)}$, the analogue of $T_{3}^{(1)}$, reads exactly as $T_{3}^{(1)}$, Eq. (22), but with all position operators interchanged with their corresponding momentum operators. It can be shown that if a pure symmetric tripartite Gaussian state with a matrix $A$ violates the inequalities for $S_{3}^{(m)}$ for some $m$ then the state with the inverse matrix $A^{-1}$ violates the inequalities for $\tilde{S}_{3}^{(m)}$ for the same $m$. In fact, we have

$$
\hat{\tilde{Z}}=-\hat{a}+\hat{b}^{\dagger}+\hat{c}^{\dagger}=-\frac{1}{\sqrt{2}}\left(\mathbf{e}^{\mathrm{T}} \mathbf{x}+\mathbf{j}^{\mathrm{T}} \nabla\right),
$$

and for the generating function $\tilde{G}(t)=\sum_{m=0}^{+\infty} \tilde{S}_{3}^{(m)} t^{m}$ we obtain the following expression:

$$
\tilde{G}(t)=\frac{1}{\sqrt{1-\frac{1+\mathbf{e}^{\mathrm{T}} A^{-1} \mathbf{e}}{2} t}} \frac{1}{\sqrt{1-\frac{1+\mathbf{j}^{\mathrm{T}} A \mathbf{j}}{2} t}} .
$$

We see that $\tilde{G}(t)$ is the same as $G(t)$ with $A \rightarrow A^{-1}$. This is a general result valid for all symmetric tripartite pure Gaussian states with real wave function.

\section{Appendix C: Exponential violation by a physical state}

For the generating function (B14) we have

$$
G(t)=\frac{1}{\sqrt{1-\alpha t}} \frac{1}{\sqrt{1-\beta t}},
$$

where

$$
\alpha=\frac{27-\sqrt{385}}{8} \approx 0.92, \quad \beta=\frac{61-\sqrt{385}}{24} \approx 1.72 .
$$


From this expression we obtain

$$
\begin{aligned}
& S_{3}^{(m)}=\frac{1}{2^{2 m}} \sum_{k=0}^{m}\left(\begin{array}{c}
2 k \\
k
\end{array}\right)\left(\begin{array}{c}
2(m-k) \\
m-k
\end{array}\right) \alpha^{k} \beta^{m-k} \\
& =\frac{\beta^{m}}{2^{2 m}} \sum_{k=0}^{m}\left(\begin{array}{c}
2 k \\
k
\end{array}\right)\left(\begin{array}{c}
2(m-k) \\
m-k
\end{array}\right)\left(\frac{\alpha}{\beta}\right)^{k}=\beta^{m} E_{m}(x),
\end{aligned}
$$

where $x=\alpha / \beta<1$. We prove that the quantity

$$
E_{m}(x)=\frac{1}{2^{2 m}} \sum_{k=0}^{m}\left(\begin{array}{c}
2 k \\
k
\end{array}\right)\left(\begin{array}{c}
2 m-2 k \\
m-k
\end{array}\right) x^{k}
$$

monotonically decreases with $m$ for any fixed $x$ provided that $0 \leqslant x<1$. Note that $E_{m}(1)=1$. In fact, we have

$$
E_{m}(1)=\left[t^{m}\right] \frac{1}{\sqrt{1-t}} \frac{1}{\sqrt{1-t}}=\left[t^{m}\right] \frac{1}{1-t}=1 .
$$

Also note that $\left(\begin{array}{c}2 n+2 \\ n+1\end{array}\right)=(4-2 /(n+1))\left(\begin{array}{c}2 n \\ n\end{array}\right)$ for $n \geqslant 0$. With these two facts established, we can now estimate $E_{m+1}(x)$ as $E_{m+1}(x)=E_{m}(x)-R_{m+1}(x)$, where

$$
\begin{aligned}
R_{m+1}(x) & =\frac{1}{2^{2 m+2}}\left[2 \sum_{k=0}^{m} \frac{\left(\begin{array}{c}
2 k \\
k
\end{array}\right)}{k+1}\left(\begin{array}{c}
2(m-k) \\
m-k
\end{array}\right) x^{k}\right. \\
& \left.-\left(\begin{array}{c}
2(m+1) \\
m+1
\end{array}\right) x^{m+1}\right] .
\end{aligned}
$$

Since $E_{m+1}(1)=E_{m}(1)=1$, we get the equality

$$
2 \sum_{k=0}^{m} \frac{1}{k+1}\left(\begin{array}{c}
2 k \\
k
\end{array}\right)\left(\begin{array}{c}
2(m-k) \\
m-k
\end{array}\right)=\left(\begin{array}{c}
2(m+1) \\
m+1
\end{array}\right) .
$$

According to our assumption, $0 \leqslant x<1$, and thus we can estimate the first part of $R_{m+1}(x)$ as follows:

$$
\begin{aligned}
& 2 \sum_{k=0}^{m} \frac{1}{k+1}\left(\begin{array}{c}
2 k \\
k
\end{array}\right)\left(\begin{array}{c}
2(m-k) \\
m-k
\end{array}\right) x^{k} \\
& >2 \sum_{k=0}^{m} \frac{\left(\begin{array}{c}
2 k \\
k
\end{array}\right)}{k+1}\left(\begin{array}{c}
2(m-k) \\
m-k
\end{array}\right) x^{m+1}=\left(\begin{array}{c}
2(m+1) \\
m+1
\end{array}\right) x^{m+1},
\end{aligned}
$$

so that $R_{m+1}(x)>0$ and thus $E_{m+1}(x)<E_{m}(x)$.

We now prove that the state $\left|\varphi_{a}\right\rangle$ with $a=1.5$ violates the inequalities for $S_{3}^{(m)}$ for all $m \geqslant 1$. We must prove that $\beta^{m} E_{m}(\alpha / \beta)<\left(2^{m}+2\right) / 3$ for all $m$. For $m=1,2,3$ it is easy to prove this inequality by hand. For example, for $m=1$ it reads as $(71-2 \sqrt{385}) / 24<4 / 3$, which can be equivalently transformed to the inequality $39<2 \sqrt{385}$ and finally to the valid inequality $1521<1540$. The other two cases can be established in a similar way, with integer arithmetic only. To prove the inequality for $m>3$ note that it is enough to prove a more strict inequality

$$
c \beta^{m}<\frac{2^{m}+2}{3}, \quad c=E_{1}\left(\frac{\alpha}{\beta}\right) .
$$

If for some $m$ we show that $c(\beta-1) \beta^{m}<2^{m} / 3$ or, equivalently, that $3 c(\beta-1)<(2 / \beta)^{m}$, then the validity of the inequality $\mathrm{C} 8$ for $m$ will imply its validity for $m+1$. It is possible to show that the inequality $3 c(\beta-1)<(2 / \beta)^{m}$ holds true for $m=4$ and thus for all $m \geqslant 4$. We see that the violation grows as $(2 / \beta)^{m} \approx 1.16^{m}$. This violation grows at a more moderate rate than that of the unphysical limit presented before, but it is attained for a real physical state.
[1] A. C. Doherty, P. A. Parrilo, and F. M. Spedalieri, Phys. Rev. A 69, 022308 (2004).

[2] A. Peres, Phys. Rev. Lett. 77, 1413 (1996).

[3] M. Horodecki, P. Horodecki, and R. Horodecki, Phys. Lett. A 223, 1 (1996).

[4] R. F. Werner and M. M. Wolf, Phys. Rev. Lett. 86, 3658 (2001).

[5] R. Simon, Phys. Rev. Lett. 84, 2726 (2000).

[6] G. Giedke, B. Kraus, M. Lewenstein, and J. I. Cirac, Phys. Rev. A 64, 052303 (2001).

[7] P. van Loock and A. Furusawa, Phys. Rev. A 67, 052315 (2003).

[8] T. Aoki, N. Takei, H. Yonezawa, K. Wakui, T. Hiraoka, A. Furusawa, and P. van Loock, Phys. Rev. Lett. 91, 080404 (2003).

[9] L.-M. Duan, G. Giedke, J. I. Cirac, and P. Zoller, Phys. Rev. Lett. 84, 2722 (2000).

[10] L. K. Shalm, D. R. Hamel, Z. Yan, C. Simon, K. J. Resch, and T. Jennewein, Nat. Phys. 9, 19 (2013).

[11] P. Hyllus and J. Eisert, New Journal of Physics 8, 51 (2006).

[12] J. Sperling and W. Vogel, Phys. Rev. Lett. 111, 110503
(2013).

[13] A. Acín, D. Bruß, M. Lewenstein, and A. Sanpera, Phys. Rev. Lett. 87, 040401 (2001).

[14] S. L. Braunstein, Phys. Rev. A 71, 055801 (2005).

[15] M. Reck, A. Zeilinger, H. J. Bernstein, and P. Bertani, Phys. Rev. Lett. 73, 58 (1994).

[16] G. Adesso and S. Piano, Phys. Rev. Lett. 112, 010401 (2014).

[17] S. Olivares and M. G. Paris, Eur. Phys. J. Special Topics 160, 319 (2008).

[18] E. Shchukin and W. Vogel, Phys. Rev. A 74, 030302 (2006).

[19] E. Shchukin and W. Vogel, Phys. Rev. Lett. 96, 200403 (2006).

[20] A. Allevi, S. Olivares, and M. Bondani, Phys. Rev. A 85, 063835 (2012).

[21] H. Nha, S.-Y. Lee, S.-W. Ji, and M. S. Kim, Phys. Rev. Lett. 108, 030503 (2012).

[22] There is an alternative way of proving this theorem that does not use PT explicitly. But nevertheless it turns out that the theorem is PT-related - if a state is PPT then the inequality 2 cannot be violated. 
[23] G. Adesso, A. Serafini, and F. Illuminati, Phys. Rev. A 73, 032345 (2006).

[24] W. P. Bowen, P. K. Lam, and T. C. Ralph, J. Mod. Opt 50, 801 (2003).

[25] P. van Loock and S. L. Braunstein, Phys. Rev. Lett. 84, 3482 (2000).

[26] M. S. Kim, J. Lee, and W. J. Munro, Phys. Rev. A 66,
030301 (2002).

[27] L. Mandel and E. Wolf, Optical coherence and quantum optics (Cambridge, 1995) p. 525, Eq. (11.3-4a)

[28] A. Erdélyi, ed., Higher transcendental functions, Vol. 2 (1953) p. 194, Eq. (22)

[29] R. A. Horn and C. R. Johnson, Matrix analysis, 2nd ed. (Cambridge, 2013) p. 26, Eq. (0.8.5.11) 